\title{
El marco curricular de la Educación Secundaria Obligatoria: posibilidades para la formación de competencias en sostenibilidad
}

The Curriculum Framework of Compulsory Secondary Education: possibilites for training skill in sustainability

- María Patta Tomás y María Ángeles Murga Menoyo

Universidad Nacional de Educación a Distancia, España

$\left(\begin{array}{l}\text { Fecha de recepción: } 15 \text { de octubre de } 2020 \\ \text { Fecha de aprobación: } 26 \text { de noviembre de } 2020\end{array}\right)$

DOI: http://dx.doi.org/10.15304/ricd.3.13.7180

\section{NOTAS BIOGRÁFICAS}

María Patta Tomás pertenece al Cuerpo de Profesoras de Educación Secundaria y es Investigadora Predoctoral de la Escuela Internacional de Doctorado de la UNED. Licenciada en Biología y en Ciencias Políticas y Sociología y doctora en Biología. Ejerce su función docente en el Instituto de Enseñanza Secundaria de Noreña (Asturias) impartiendo las asignaturas de Biología y Geología y Cultura Científica en Educación Secundaria Obligatoria y Bachiller.

Contacto: esdelosmares@gmail.com

María Ángeles Murga Menoyo es profesora titular del Departamento de Teoría de la Educación y Pedagogía Social de la UNED. Miembro de la Cátedra Unesco de Educación Ambiental y Desarrollo Sostenible, es investigadora principal del proyecto actualmente en curso: La alfabetización ecosocial: un elemento central en los procesos de sostenibilización curricularpara el logro de la Agenda 2030 (ODS) en la formación inicial del profesorado (PRAD-ODS), financiado por Ministerio de Ciencia, Innovación y Universidades (2019-2021). Coordina el Programa de Doctorado en Educación de la UNED.

Contacto:mmurga@edu.uned.es

\section{Resumen}

Los Objetivos de Desarrollo Sostenible (ODS) hacen necesaria la formación de competencias en sostenibilidad, para lo cual se requiere que los currículos de la Educación Secundaria Obligatoria incluyan como meta educativa la formación de este tipo de competencias. El propósito de este trabajo es analizar la coherencia entre las competencias exigidas por el currículo oficial de la asignatura Biología y Geología de 1ํo de ESO (RD 1105/2014, de 26 de diciembre) y las competencias necesarias para la consecución de la Agenda 2030 de las Naciones Unidas. Se realiza un análisis comparativo de los elementos que conforman ambas categorías de competencias y se identifica su presencia explicita en el marco curricular de la asignatura. Los resultados muestran la ausencia en el temario oficial de los conceptos de sistema, sostenibilidad y desarrollo sostenible, significativos en el campo de las dos disciplinas, y un déficit acusado de la competencia sistémica que afecta a la adquisición de todas las demás, tanto las necesarias para el desarrollo sostenible como las más tradicionales requeridas en los currículos oficiales. El efecto es un alumnado formado para comprender conceptos, hechos y situaciones aisladas, aunque coninsuficiente conocimiento de las interrelaciones que se producen en 
la realidad ecosocial, cuestión imprescindible para el logro de los ODS. Se constata la necesidad de reorientar la práctica docente hacia modos de hacer que impliquen no solo profundizar en el enfoque de la educación por competencias, reequilibrando el actual predominio de los contenidos sobre sus restantes componentes, sino también que fundamenten la acción en un marco de valores y actitudes coherentes con las necesidades y demandas sociales más urgentes ante la crisis ambiental. Este propósito requiere articular el proceso de enseñanza-aprendizaje con un enfoque metodológico que apueste por integrar conocimientos y emociones, a través de la propia experiencia de interacción con el contexto.

\section{Abstract}

The Sustainable Devel opment Goals (SDG) require training of sustainability competencies, for which it is required that the curricula of Compulsory Secondary Education include the training of this type of competencies as an educational goal. The purpose of this study is to analyze the coherence between the competencies required by the official curriculum of the Biology and Geology in the first year of Secondary School (RD 1105/2014, December 26) and the key competencies for the achievement of the UN 2030 Agenda. A comparative analysis of the elements that make up both categories of competencies is carried out and their explicit presence in the curricular framework of the subject is identified. The results show the absence in the official agenda of the concepts of system, sustainability and sustainable development, significant in the field of the two disciplines, and a marked deficit of systemic competition that affects the acquisition of all the others, both, those necessary for sustainable development and the more traditional ones required in the official curricula. The effect is a student trained to understand isolated concepts, facts and situations, although with insufficient knowledge of the interrelationships that occur in the eco-social reality, an essential issue for the achievement of the SD Gs. The need to redirect teaching practice towards ways of doing that imply not only deepening the focus of education by competencies, rebalancing the current predominance of the contents over its other components, butalso that they base the action on a framework of values and attitudes consistent with the most urgent social needs and demands in the face of the environmental crisis. This purpose requires articulating the teaching-learning process with a methodological approach that is committed to integrating knowledge and emotions, through the experience of interaction with the context.

\section{Palabras clave}

Educación para el desarrollo sostenible, sostenibilización curricular, educación secundaria, competencias en sostenibilidad, práctica docente.

\section{Keywords}

Education for sustainable development, curricular sustainability, secondary education, sustainability skills, teaching practice. 


\section{Sumario}

1. Introducción

2. Clarificación conceptual

2.1. Competencias clave para los ODS: las competencias en sostenibilidad

2.2. Tipología de las competencias clásicas: el modelo educativo dominante

2.3. Competencias en sostenibilidad versus competencias clásicas

3. Análisis del marco curricular de la asignatura Biología y Geología de 1o de ESO

3.1. Objetivos y procedimiento

\subsection{Resultados}

\subsubsection{Contenidos y estándares de aprendizaje: contribución a los ODS}

3.2.2. Capacidades que la asignatura forma relacionadas con las competencias en sostenibilidad

3.2.3. Contribución de la asignatura Biología y Geología de $1^{\circ}$ de ESO a la formación de competencias para los ODS

4. Conclusiones

\section{Summary}

1. Introduction

2. Conceptual clarification

2.1. Key competencias for the SDGs: sustainability competencies

2.2. Typology of classical competencias: the dominant educational model

2.3. Sustainability competencias versus classical competencias

3. Analysis of the curricular framework of Biology and Geology in the first year of Secondary School

3.1. Objectives and procedure

3.2. Results

3.2.1. Content and learningstandards: contribution to the SDGs

\subsubsection{Capacities that the subject forms related to sustainability competencias}

3.2.3. Contribution of Biology and Geology in the First Year of Secondary School to the training of competencies for the SDGs

4. Conclusions 


\section{INTRODUCCIÓN}

La crisis ecológica del planeta, puesta de manifiesto por indicadores como el cambio climático, el deterioro de la biodiversidad, la contaminación del aire, del agua y del suelo, la desigualdad y la pobreza (ONU, 2017; PNUD, 2018; WWF, 2020), señala la necesidad de un cambio en los patrones sociales de producciónconsumo y, para ello, en los estilos de vida dominantes y los valores que los sustentan. Naciones Unidas, ante tamaño reto, impulsó, en 2015, un plan de acción en favor de las personas, el planeta y la prosperidad, conocido como Agenda 2030 (ONU, 2015). Este programa comprende 17 objetivos (ODS) con 169 metas, que son de aplicación universal y que implican a todas las personas y países. Su consecución requiere reorientar la educación hacia el desarrollo de competencias que no parecen suficientemente contempladas en el actual modelo educativo, en cuya base está el Proyecto de Definición y Selección de Competencias (DeSeCo) promovido por la Organización para la Cooperación y el Desarrollo Económico (OCDE, 2005).

Siguiendo este modelo, en España, la LOMCE, Ley Orgánica 8/2013, de 9 de diciembre, para la Mejora de la Calidad Educativa (2013) exige la formación en las aulas de las siguientes competencias clave: competencia lingüistica, competencia matemática y competencias básicas en ciencia y tecnología, digital, aprender a aprender, competencias sociales y cívicas, sentido de la iniciativa y espíritu emprendedory de conciencia y expresiones culturales.

No obstante, hace más de un lustro que UNESCO llama la atención sobre la necesidad de formar competencias específicas para la sostenibilidad y solicita al sistema educativo que cumpla esta función, ineludible para alcanzar los Objetivos de Desarrollo Sostenible. Considera competencias clave para la sostenibilidad las siguientes: análisis crítico, pensamiento sistémico, toma de decisiones colaborativay sentido de la responsabilidad hacia las generaciones presentes y futuras (UNESCO, 2014). En una revisión posterior fueron añadidas las competencias de anticipación, normativa, estratégica, de autoconciencia e integrada para la resolución de problemas (UNESCO, 2017).

Ante esta situación, nos preguntamos en qué medida el actual currículo oficial de la educación secundaria está facilitando la formación de los conocimientos, valores, actitudes, hábitos, etc. que conforman las competencias en sostenibili- dad que Unesco demanda. Porque, si bien en el Real Decreto 1105/2014, de 26 de diciembre, por el que se establece el currículo básico de la Educación Secundaria Obligatoria y del Bachillerato (2014) que recoge el currículo básico oficial vigente, no se mencionan explícitamente con el mismo nombre que tienen asignado en la literatura sobre educación para el desarrollo sostenible, cabe pensar que el marco curricular previsto, al menos en parte, podría facilitar su formación.

El estudio que a continuación se expone pretende contribuir a clarificar este interrogante, desvelando lascoincidencias entre los elementos de las competencias consideradas básicas para la sostenibilidad y aquellos que conforman las competencias clave (en adelante "competencias clásicas') que la normativa vigente establece como obligatorias. El foco de atención se sitúa en la asignatura de Biología y Geología de $1^{\circ}$ de la ESO, con la finalidad de conocer las posibilidades que brinda el marco curricular de la asignatura para una práctica docente de calidad que pueda contribuir al desarrollo sostenible.

Tras esta introducción, se aborda, en primer lugar, la clarificación conceptual y la definición operativa, mediante la revisión de la literatura publicada, de las cuatro competencias clave para la sostenibilidad que Unesco propuso inicialmente (UNESCO, 2014), considerando que en ellas se encuentra en síntesis la formación básica que la sostenibilidad requiere en el nivel de la educación secundaria obligatoria, objeto de nuestro estudio. A continuación, se presentan las competencias clásicas, sus elementos y componentes más significativos, $y$, fruto del análisis realizado, un primer resultado del estudio, las similitudes y diferencias entre las que hemos denominado competencias clásicas y las competencias en sostenibilidad. En tercer lugar, se describen los objetivos, procedimientos y resultados del análisis del marco curricular oficial de la asignatura objeto de estudio. El articulo finaliza con la exposición de conclusiones.

\section{CLARIFICACIÓN CONCEPTUAL}

\subsection{COMPETENCIAS CLAVE PARA LOS ODS: LAS COMPETENCIAS EN SOSTENIBILIDAD}

La formación de competencias es la respuesta arbitrada por el modelo educativo dominante ante la necesidad de aprendizaje permanente de una ciudadanía abocada a desarrollar su vida en contextos que cambian con mucha rapidez 
(Consejo de la Unión Europea, 2018). El témino competencia, sobre el que existe una amplia literatura publicada (Rychen, Salganik, Moser y Konstant, 2000; Rychen y Salganik, 2004; OCDE, 2005; entre otros), es un concepto multidimensional que implica conocimientos, habilidades, destrezas, actitudes y valores que se traducen en una formación integral del alumnado, susceptible de evaluación. En este contexto, el Parlamento Europeo y el Consejo de Europa (2006) adoptaron el marco de competencias clave que los ciudadanos necesitan para la participación democrática en los asuntos sociales y económicos, la cohesión social, la empleabilidad y como contexto para la toma de decisiones políticas e institucionales, y solicitaron su abordaje en los programas educativos y de formación. Este planteamiento se contempla así mismo como base formativa en relación con el ODS 4 de la Agenda 2030 (ONU, 2015), que reclama no solo una educación de calidad sino también una educación inclusiva y equitativa, en el marco del aprendizaje a lo largo de la vida (Comisión Europea, 2015).

En el año 2014, la Conferencia General de la UNESCO aprobó el Programa Mundial para el Desarrollo Sostenible en el cual se destacaba la necesidad de iniciar procesos de sostenibilización curricular para integrar los principios y valores de la sostenibilidad en el modelo educativo (UNESCO, 2014). Esta propuesta solicitaba la inclusión de conocimientos sobre los problemas medioambientales y la formación de nuevas competencias entre los objetivos formativos de los currículos de todos los niveles del sistema educativo, con la finalidad de que los alumnos y alumnas pudieran afianzar los comportamientos y valores necesarios para analizar las informaciones, relacionar hechos y, en la medida de sus posibilidades, tomar decisiones a favor del entorno así como intervenir en su mantenimiento por propia iniciativa o en colaboración con otros grupos de interés.

La tabla 1 recoge las definiciones y los elementos de las cuatro competencias esenciales que inicialmente Unesco (2014) propuso para orientar el cambio hacia el desarrollo sostenible. La primera de ellas, el pensamiento crítico, plantea a la educación el reto de formar seres humanos independientes y autónomos, capaces de buscar la verdad por sí mismos (Richard y Elder, 2005), más allá de sesgos de interés, presentaciones espurias, ocultaciones intencionales o lagunas reales de conocimiento; y hacerlo dentro de un marco ético que les permita asumir una posición propia y generar interpretaciones y emociones que lleven a la acción.

Para ello se requiere que desarrollen la capacidad de indagar sobre todas las cosas, situaciones y problemas, a fin de detectar posibles sesgos o problemas, a la vez que ejercitan la reflexión, la flexibilidad, la curiosidad y la motivación (UNESCO, 2014). Estas capacidades son características de las personas autocríticas, no egocéntricas, habituadas a profundizar en los temas buscando sus causas y consecuencias (Richard y Elder, 2005). Todo ello es condición necesaria como base de la capacidad para analizar los problemas de desarrollo sostenibley actuar en consecuencia (Wiek,Withycombe \& Redman, 2011).

Por su parte, la competencia de pensamiento sistémico permite la comprensión holística tras el análisis de conjunto de los sistemas complejos en diferentes dominios y escalas, teniendo en cuenta que todo elemento se encuentra en constante interacción con otros elementos del sistema, al igual que con el sistema mismo (Morín, 2004). Y los comportamientos a favor del desarrollo sostenible no son posibles sin que el alumnado comprenda que toda acción tiene repercusiones de alcance, más allá del lugar donde se genera, y que, en consecuencia, cada persona forma parte de un sistema sobre el que actúa mediante su estilo de vida y sobre el que puede actuar de forma intencionada.

La competencia sistémica permite conclusiones derivadas de la percepción integrada de todos los elementos del entorno y pasar de la descripción a la interpretación del contexto (Novo, Mandón y Marpegán, 2002). Adquirir la competencia sistémica facilita a la persona la posibilidad de analizar sistemas complejos, de carácter social, económico, medioambiental, etc: tanto en el nivel local como global (Wiek, Withycombe \& Redman, 2011). 
Tabla 1: Definición de las competencias clave para el desarrollo sostenible

\begin{tabular}{|l|l|}
\hline COMPETENCIA & DEFINIClÓN \\
\hline PENSAMIENTO & $\begin{array}{l}\text { Es el proceso de analizary evaluar el pensamiento con el propósito de } \\
\text { mejorarlo y emplearlo en el razonamiento a través de problemas y } \\
\text { asuntos reales (1). Este pensamiento permite analizar cualquier } \\
\text { información, con el fin de valorarla objetivamentey eliminar los posibles } \\
\text { sesgos que pueda contener que comprometen la objetividad de los } \\
\text { datos investigados (6) y tomar decisiones responsables para el } \\
\text { desarrollo sostenible (2). }\end{array}$ \\
\hline $\begin{array}{l}\text { PENSAMIENTO } \\
\text { SISTÉMICO }\end{array}$ & $\begin{array}{l}\text { global y holística que atraviesa dominios temporales, espaciales y } \\
\text { conceptuales, donde el todo, más allá de la suma de las partes, tiene } \\
\text { cualidades emergentes, Interrelaciones e incertidumbres" (3). Otorga la } \\
\text { habilidad de "analizar conjuntamente complejos sistemas que }\end{array}$ \\
atraviesan diferentes dominios (sociedad, medioambiente, economía, \\
etc.) a través de diferentes escalas (de local a global), considerando a \\
su vez una cascada de efectos, inercia, lazos de retroalimentación y \\
otras características sistémicas relacionadas con cuestiones de \\
sostenibilidad y marcos de resolución de problemas" (2).
\end{tabular}

Fuente: Elaboración propia a partir de autores varios: (1) Richard y Elder, 2005; (2) Wiek Withycombe \& Redman, 2011, p. 5-9; (3) Morin, 2010; (4) Kozlowski \& Ilgen, 2006; (5) Murga-Menoyo, 2015; (6) UNESCO, 2014.

Una tercera competencia clave para avanzar en la sostenibilidad es la toma de decisiones colaborativa, cuya necesidad tiene sus raíces en los principios democráticos más elementales. Y para su florecimiento, teniendo en cuenta que las decisiones a favor de los objetivos de desarrollo sostenible implican a personas de diferentes contextos e intereses, resulta esencial trabajar en las relaciones interpersonales. El alumnado debe desarrollar habilidades argumentativas, participativas, de responsabilidad individual y grupal y compromiso democrático y con los Derechos Humanos, con el fin de integrarse con éxito en grupos de trabajo que propicien la ayuda mutua en la resolución de problemas. Debe ser, asimismo, capacitado para comprender la responsabilidad que tiene por las consecuencias de sus actos, no solo sobre las generaciones presentes sino también sobre las que aún no existen; $y$, a la vez, para ser consciente de su posible contribución al cambio por la sostenibilidad, adoptando alternativas disponibles a los estilos de vida injustos e insostenibles hoy consolidados.

Finalmente, la competencia responsabilidad con las generaciones presentes y futuras busca que las generaciones actuales tomen plena conciencia de la responsabilidad de sus acciones, por sus efectos actuales y por la repercusión futura sobre la especie humana, yse comprometan activamente para salvaguardar la dignidad de las personas y sus necesidades; también a preservar todos los bienes materialese inmateriales, tales como el genoma humano, la diversidad biológica y cultural, el Patrimonio Común de la Humanidad, la educación y la paz. Este propósito 
implica desarrollar el pensamiento crítico, la compasión y el compromiso ético y social para prevenir y contrarrestar los posibles escenarios presentes y futuros que no garanticen los derechos humanos de todas las personas (UNESCO, 2014).

\subsection{TIPOLOGÍA DE LAS COMPETENCIAS CLÁSICAS: EL MODELO EDUCATIVO DOMINANTE}

Las competencias clásicas, centrales en el modelo educativo dominante, fueron establecidas para la formación integral de un alumnado que necesitaba adaptarse a una diversidad creciente de contextos académicos, sociales y profesionales. Cada una de ellas se encuentra definida en el proyecto DeSeCo (OCDE, 2005) y, posteriormente, específicamente para el caso que nos ocupa, en la Orden ECD/65/2015, de 21 de enero, por la que se describen las relaciones entre las competencias, los contenidos y los criterios de evaluación de la educación primaria, la educación secundaria obligatoria y el bachillerato (2015).

Como se recoge en la tabla 2 , tenemos, en primer lugar, la competencia lingüística, necesaria para articular un discurso coherente en diversas situaciones. Requiere el dominio de la corrección gramatical y semántica, de un vocabulario adecuado a la situación y una correcta expresión oral y escrita. Otras dos competencias clave -matemática y básica de ciencia y tecnología- se fundamentan en conceptos como cantidad, forma, espacio, relaciones o incertidumbre, y proporcionan al alumnado herramientas para interpretar conceptos, datos, procedimientos y relaciones y aplicarlos a la resolución de situaciones propias de la vida cotidiana, personal y social. A través de la adquisición de la competencia digital se pretende la alfabetización digital y el desarrollo de la capacidad de manejar la información procedente de entornos virtuales en condiciones de seguridad para obtener conocimiento útil; asimismo, se propicia que se puedan utilizar diversas herramientas propias de las infotecnologías para el aprendizaje y la comunicación.

Una cuarta competencia clásica es la competencia de aprender a aprender en cuya base se encuentran elementos como la motivación, la planificación, la persistencia y la comunicación, que permiten a la persona obtener por sí misma el conocimiento que necesita y aplicarlo a todos los aspectos de su vida. También resultan imprescindibles tanto la competencia social y cívica como la competencia de conciencia y expresiones culturales; ambas persiguen la integración del alumnado en la sociedad, desde su propia identificación personal y el respeto por los derechos humanos, la diversidad de manifestaciones culturales y la normativa, comprendiendo la heterogeneidad y la complejidad de esta. Por último, la competencia de iniciativa y espíritu emprendedor e innovación prepara al alumnado para la adopción de decisiones autónomas y la asunción de riesgos e incertidumbres inherentes a la transformación de ideas en actos.

Tabla 2. Definición de las competencias clásicas

\begin{tabular}{|c|c|}
\hline COMPETENCIA & DEFINICIÓN \\
\hline LINGÜÍSTICA & $\begin{array}{l}\text { “La competencia en comunicación lingüística (CCL) es el resultado de la acción } \\
\text { comunicativa dentro de prácticas sociales determinadas, en las cuales el } \\
\text { individuo actúa con otros interlocutores y a través de textos en múltiples } \\
\text { modalidades, formatosysoportes”. }\end{array}$ \\
\hline $\begin{array}{l}\text { MATEMÁTICAY } \\
\text { B. EN CIENCIAY } \\
\text { TECNOLOGÍA }\end{array}$ & $\begin{array}{l}\text { "La competencia matemática implica la capacidad de aplicar el razonamiento } \\
\text { matemático y sus herramientas para describir, interpretar y predecir distintos } \\
\text { fenómenos en su contexto". } \\
\text { "Las competencias básicas en ciencia y tecnología son aquellas que proporcionan } \\
\text { un acercamiento al mundo físico y a la interacción responsable con él desde } \\
\text { acciones, tanto individuales como colectivas, orientadas a la conservación y } \\
\text { mejora del medio natural, decisivas para la protección y mantenimiento de la } \\
\text { calidad de vida y el progreso de los pueblos" (CMCT). }\end{array}$ \\
\hline DIGITAL & $\begin{array}{l}\text { "La competencia digital(CD) es aquella que implica el uso creativo, críticoy seguro } \\
\text { de las tecnologías de la información y la comunicación para alcanzar los objetivos } \\
\text { relacionados con el trabajo, la empleabilidad, el aprendizaje, el uso del tiempo } \\
\text { libre, la inclusión y participación en la sociedad". }\end{array}$ \\
\hline
\end{tabular}




\begin{tabular}{|l|l|}
\hline $\begin{array}{l}\text { APRENDER A } \\
\text { APRENDER }\end{array}$ & $\begin{array}{l}\text { "La competencia para a prender a a prender (CPAA). Supone la habilidad para } \\
\text { iniciar, organizary persistir en el aprendizaje. }\end{array}$ \\
\hline SOCIALY CIVICA & $\begin{array}{l}\text { "Las competencias sociales y cívicas conllevan la habilidad y capacidad para } \\
\text { utilizar los conocimientos y actitudes sobre la sociedad, entendida desde las } \\
\text { diferentes perspectivas, en su concepción dinámica, cambiante y compleja, para } \\
\text { interpretarfenómenos y problemas sociales". }\end{array}$ \\
\hline $\begin{array}{l}\text { INICIATIVAY } \\
\text { ESPÍRITU } \\
\text { EMPRENDEDOR }\end{array}$ & $\begin{array}{l}\text { "La competencia sentido de la in iciativa y espíritu emprendedor (SIE) para } \\
\text { transformar las ideas en actos". }\end{array}$ \\
\hline $\begin{array}{l}\text { CONCIENCIAY } \\
\text { EXPRESIONES } \\
\text { CULTURALES }\end{array}$ & $\begin{array}{l}\text { "La competencia en conciencia y expresiones culturales (CEC) implica conocer, } \\
\text { comprender, apreciar y valorar con espíritu crítico, con una actitud abierta y } \\
\text { respetuosa, las diferentesmanifestaciones culturales yartísticas, utilizarlas como } \\
\text { fuente de enriquecimiento y disfrute personal y considerarlas como parte de la } \\
\text { riqueza y patrimonio de los pueblos". }\end{array}$ \\
\hline
\end{tabular}

Fuente: Orden ECD/65/2015, de 21 de enero, por la que se describen las relaciones entre las competencias, los contenidos y los criterios de evaluación de la educación primaria, la educación secundaria obligatoria y el bachillerato (2015)

En cuanto a los elementos que conforman estas ocho competencias clásicas, en la tabla 3 se explicitan sus componentes y de las capacidades que implican; todas ellas contribuyen a la formación integral del alumnado para su participación plena en la sociedad.

Por una parte, para consolidar un análisis y pensamiento crítico se requiere el desarrollo de las competencias matemática y de ciencia y tecnología, así como la capacidad de comunicar el pensamiento mediante discursos coherentes con la situación y el entorno que proporciona la competencia lingüística. Por otra, la competencia de aprender a aprender para planificar las nece- sidades de aprendizaje a partir de uno mismo y sus propias herramientas, incluidas las que aportan las nuevas tecnologías mediante la competencia digital. También se espera que el alumnado sea capaz de abordar situaciones, necesidades e intereses para plantear sus propias soluciones, capacidades que forma la competencia de iniciativa y espíritu em prendedor. Por último, las competencias sociales y cívica y de conciencia y expresiones culturales buscan formara un ciudadano que participe de forma democrática en la sociedad, utilizando los códigos de conducta pertinentes, en el respeto de los derechos humanos y de la diversidad cultural y artística.

Tabla 3. Componentes y capacidades de las competencias clásicas

\begin{tabular}{|c|c|c|}
\hline COMPETENCIA & COMPONENTES & CAPACIDADES \\
\hline LINGÜISTICA & $\begin{array}{l}\text {-Léxica } \\
\text {-Gramatical } \\
\text {-Semántica } \\
\text {-Fonológica } \\
\text {-Ortográfica }\end{array}$ & $\begin{array}{l}\text {-Expresary estructurar pensamientos, } \\
\text { emociones, vivencias y opiniones. } \\
\text {-Dar coherencia y cohesión al discurso ya las } \\
\text { propias acciones y tareas } \\
\text {-Establecer vínculos y relaciones constructivas } \\
\text { con los demás y con el entorno en diferentes } \\
\text { contextos. } \\
\text {-Interpretary comprender la realidad. }\end{array}$ \\
\hline $\begin{array}{l}\text { MATEMÁTICAYB. EN } \\
\text { CIENCIAY } \\
\text { TECNOLOGÍA }\end{array}$ & $\begin{array}{l}\text {-Cantidad } \\
\text {-Espacio y forma } \\
\text {-Cambio y relaciones } \\
\text {-Incertidumbre y datos } \\
\text {-Sistemas físicos, } \\
\text { biológicos, de la Tierra y } \\
\text { el espacio y tecnológicos }\end{array}$ & $\begin{array}{l}\text {-Interpretar resultados matemáticos de acuerdo } \\
\text { con el contexto. } \\
\text {-Relacionar conceptos y procedimientos en la } \\
\text { resolución de tareas. } \\
\text {-Comprender unidades, magnitudes, cálculos } \\
\text { tendencias y patrones numéricos. } \\
\text {-Comprender la perspectivay la construcción y } \\
\text { transformación de las formas. } \\
\text {-Interpretación y valoración de conclusiones en } \\
\text { situaciones de incertidumbre. }\end{array}$ \\
\hline
\end{tabular}




\begin{tabular}{|c|c|c|}
\hline & & $\begin{array}{l}\text {-Identificar, plantear y resolver situaciones de la } \\
\text { vida cotidiana, personal y social. }\end{array}$ \\
\hline DIGITAL & $\begin{array}{l}\text {-Información y } \\
\text { alfabetización } \\
\text {-Comunicación y } \\
\text { colaboración } \\
\text {-Creación de contenido } \\
\text {-Seguridad online } \\
\text {-Resolución de } \\
\text { problemas }\end{array}$ & $\begin{array}{l}\text {-Transformar la información digital en } \\
\text { conocimiento útil. } \\
\text {-Adecuarse a los cambios impuestos por las } \\
\text { nuevas tecnologías. } \\
\text {-Valorar críticamente las fuentes de } \\
\text { información. } \\
\text {-Trabajar con seguridad en el marco digital. } \\
\text {-Resolver problemas realesa partir de } \\
\text { herramientas digitales. }\end{array}$ \\
\hline $\begin{array}{l}\text { APRENDER A } \\
\text { APRENDER }\end{array}$ & $\begin{array}{l}\text {-Requerimientos de la } \\
\text { tarea } \\
\text {-Motivación } \\
\text {-Planificación, } \\
\text { persistencia, evaluación } \\
\text {-Comunicación } \\
\text {-Trasferencia a otras } \\
\text { situaciones. }\end{array}$ & $\begin{array}{l}\text {-Organizar y gestionar el propio aprendizaje } \\
\text { conociendo los propios procesos mentales. } \\
\text {-Mantener la motivación y la confianza. } \\
\text {-Comprender las necesidades de la tarea. } \\
\text {-Asimilar nuevos conocimientos y dominar } \\
\text { capacidades y destrezas de un ámbito } \\
\text {-Solucionar problemas a partir de sus propias } \\
\text { herramientas. }\end{array}$ \\
\hline SOCIALY CIVICA & $\begin{array}{l}\text {-Identificación individual } \\
\text { y social } \\
\text {-Ciudadanía e } \\
\text { integración social } \\
\text {-Derechos humanos } \\
\text {-Normativa }\end{array}$ & $\begin{array}{l}\text {-Participar plenamentey de forma democrática } \\
\text { en la vida cívica y social. } \\
\text {-Empatizar y aceptar las diferencias en valores, } \\
\text { creencias, cultura y situación personal o } \\
\text { colectiva. } \\
\text {-Adoptar un estilo de vida saludable para sí } \\
\text { mismo y por otras personas. } \\
\text {-Comprender los códigos de conducta y los } \\
\text { usos aceptados por las distintas sociedades. }\end{array}$ \\
\hline $\begin{array}{l}\text { INICIATIVAY } \\
\text { ESPÍRITU } \\
\text { EMPRENDEDOR }\end{array}$ & $\begin{array}{l}\text {-Autonomía e iniciativa } \\
\text {-Innovación } \\
\text {-Adaptación al cambio } \\
\text {-Asunción de riesgos } \\
\text {-Gestión de } \\
\text { incertidumbre }\end{array}$ & $\begin{array}{l}\text {-Descubrir nuevas oportunidades de } \\
\text { intervención o trabajo para sí mismo y para } \\
\text { otros. } \\
\text {-Tomar conciencia de la situación de inicio, } \\
\text { aportar soluciones creativas y gestionar el } \\
\text { riesgo y la incertidumbre. } \\
\text {-Conocer los intereses de los interlocutores, } \\
\text { saber negociare implicar a otras personas. } \\
\text { Tomar decisiones con todas sus } \\
\text { consecuencias. }\end{array}$ \\
\hline $\begin{array}{l}\text { CONCIENCIAY } \\
\text { EXPRESIONES } \\
\text { CULTURALES }\end{array}$ & $\begin{array}{l}\text {-Emociones } \\
\text {-Respeto a la diversidad } \\
\text {-Imaginación y } \\
\text { creatividad } \\
\text {-Sentido estético } \\
\text {-Libertad de expresión }\end{array}$ & $\begin{array}{l}\text {-Conocer, comprender, apreciar y valorar } \\
\text { críticamente diferentes manifestaciones } \\
\text { culturales y artísticas. } \\
\text {-Apreciar las manifestaciones culturales y } \\
\text { artísticas de la vida cotidiana. } \\
\text {-Considerar las manifestaciones culturales y } \\
\text { artísticas como fuente de enriquecimiento y } \\
\text { disfrute y patrimonio de los pueblos. }\end{array}$ \\
\hline
\end{tabular}

Fuente: elaboración propia, a partir de OECD (2005)

\subsection{COMPETENCIAS EN SOSTENIBILIDAD VERSUS COMPETENCIAS CLÁSICAS}

La comparación entre los componentes de ambos tipos de competencias pone de manifiesto los solapamientos, semejanzas y reciprocas interrelaciones. Se describirán a continuación utilizando como eje articulador las competencias en sostenibilidad

\section{A. Competencia en sostenibilidad: Pensamien- to Crítico}

Esta competencia facilita a la persona la comprensión, la organización y la expresión de ideas que cumplen con los estándares intelectuales de claridad, exactitud, precisión, relevan- 
cia, profundidad, amplitud, lógica, importancia y justicia (Richard y Elder, 2005), elementos que, a su vez, configuran las competencias matemática, científica, tecnológica, lingüística y digital. Por otra parte, las competencias de aprender a aprender e iniciativa y espíritu emprendedor aportan motivación, organización, creatividad, persistencia, manejo de la incertidumbre y coraje para transformar ideas en actos, todos elementos necesarios para un pensamiento crítico robusto. Finalmente, dos competencias: social y cívica, y conciencia y expresiones culturales, proporcionan el marco ético y emocional que referencia el pensamiento crítico. Existe una relación directa entre el desarrollo de las competencias clásicas y la formación del pensamiento crítico del alumnado.

B. Competencia en sostenibilidad: Pensamiento Sistémico

El pensamientosistémicofacultaal alumnado para interpretar la realidad desde un enfoque holístico, interactivoy atento a las emergencias e incertidumbres, propio de la complejidad cultural, social, económica y ecológica. Las competencias clásicas aportan elementos esenciales en este marco, si bien descuidan el posicionamiento e integración en el sistema y la visión global. Y tampoco contemplan explícitamente que, para la integración social y el respeto a los derechos humanos, es preciso no solo tener en cuenta el contexto sino también el impacto del estilo de vida propio sobre los recursos naturales y los restantes seres vivos. En consecuencia, las competencias clásicas adolecen de eficacia para comprender la crisis eco-social y con ello se merma la potencialidad de la iniciativa y el espíritu emprendedory, en general, de todas las competencias esenciales, consideradas clave para el desarrollo sostenible.

C. Competencia en sostenibilidad: Toma de Decisiones Colaborativa

Esta competencia faculta al alumnado para integrarse en grupos de trabajo con objetivos comunes y perspectivas diversas, en los cuales son esenciales: el compromiso, la negociación y la gestión de conflictos. Las competencias clásicas aportan a esta competencia clave para la sostenibilidad su contribución a la formación del pensamiento crítico en todas sus manifestaciones, necesario para llegar a consensos. Y también contemplan la formación de dos competencias-social y cívica y conciencia y expresiones culturales- imprescindibles para la participación en la toma de decisiones colaborativa. Sin embargo, no se contempla explícitamente la adquisición de elementoscomo la em patía, la asertividad o la asunción de riesgos, lo que podría limitar el peso de las competencias clásicas para el logro de la sostenibilidad

C. Competencia en sostenibilidad: Responsabilidad hacia Generaciones Presentes y Futuras

Esta competencia habilita al alumnado para comprender que toda agresión al medio ambiente tiene repercusiones económicas y sociales a nivel planetario (UNESCO, 2014) y, en consecuencia, la necesidad de preservar los bienes naturales y culturales que han llegado hasta el momento actual con perspectivas de futuro, mediante un compromiso activo. De acuerdo con UNESCO (2017), es necesario integrar contenidos nuevos referidos a sostenibilidad, desarrollo sostenible, consumo sostenible, pobreza y cambio climático y exigencia de acciones que demuestren responsabilidad con la propia huella ecológica y compromiso con el cambio.

Tras la clarificación del significado, componentes y características tanto de las competencias en sostenibilidad como de aquellas que cabe considerar clásicas del enfoque por competencias dominante en los actuales sistemas educativos, tal como se recogen en la normativa vigente, en los próximos apartados la atención se focaliza en la asignatura objeto de este estudio con la intención de detectar posibles lagunas que dificulten una educación de calidad para el logro de los ODS.

\section{ANÁLISIS DEL MARCO CURRICULAR DE LA ASIGNATURA}

\subsection{OBJETIVOS Y PROCEDIMIENTO}

El Real Decreto 1105/2014, de 26 de diciembre, por el que se establece el currículo básico de la Educación Secundaria Obligatoria y del Bachillerato (2014) incluye explícitamente las competencias clásicas en el currículo de la ESO, estableciendo las capacidades que la educación debe fomentar en el alumnado de este nivel educativo; a la vez, prescribe unos contenidos y señala unos estándares de aprendizaje que permitirán evaluar su logro. En este trabajo la atención se focaliza en la asignatura Biología y Geología de $1^{\circ}$ de la ESO, con la pretensión de analizar la coherencia entre las competencias que tiene asignadas y aquellas que son necesarias para la consecución de la Agenda 2030 de las Naciones Unidas. Para ello se procederá al análisis comparativo de corte cualitativo de los elementos que conforman las competencias 
exigidas en el currículo vigente y aquellos que constituyen las competenciasclave para la sostenibilidad.

\subsection{RESULTADOS}

Este apartado recoge, como resultados del estudio, las posibilidades y limitaciones que el marco curricular oficial de la asignatura ofrece para la adquisición de las competencias en sostenibilidad. Se consideran un factor central de la capacidad formativa de la disciplina para contribuir al logro de los ODS.

\subsection{Contenidos y estándares de aprendizaje: contribución a los ods}

Los contenidos de la asignatura están articulados en siete bloques temáticos: I. Habilidades, destrezas y estrategias. Metodología científica. IILa Tierra y el Universo. III. La biodiversidad en el Planeta Tierra. IV Las personas y la salud. Promoción de la salud. V-El relieve terrestre y su evolución VI-Los Ecosistemas. VII-El proyecto de investigación (RD 1105/2014, pp. 205-210).

Nuestra experiencia de aula nos señala un perfil de alumnado que, al incorporarse por primera vez a la Educación Secundaria Obligatoria en la asignatura de Biología y Geología de $1^{\circ}$ de la ESO, posee unas nociones científicas muy básicas, como son: el concepto de célula como unidad de vida, la clasificación de los seres vivos en los cinco reinos y un principio de respeto por la ciencia como fuente de conocimiento. Asimismo, tiene nociones elementales sobre los principales órganos, aparatos y sistemas de su cuerpo, enfermedades comunes y hábitos sencillos de salud. También puede mencionar algunas formas de energía.

El temario de la asignatura permite adquirir una noción básica de los principios del Método Científico, junto con todos los elementos del entorno físico medioambiental, que se extienden al ámbito de la contaminación y la gestión de residuos y a ciertos problemas sociales y económicos derivados de los mismos. E, igualmente, están previstos para reforzar y ampliar los conocimientos sobre todos los reinos de los seres vivos, junto con la adquisición de un concepto básico de ecosistema. El alumnado debe terminar el curso con conceptos sobre los factores abióticos (geosfera, atmósfera, hidrosfera) y factores bióticos, sin la obligación de entrar en el conjunto de las relaciones que se establecen entre todos ellos.

Todos estos conocimientos son necesanios para comprender las problemáticas de los ODS 3 (Salud y bienestar), ODS 6 (Agua limpia y saneamiento), ODS 7 (Energía asequibley no contaminante), ODS 12 (Producción y consumo responsable), ODS 13 (Acción por el clima), ODS 14 (Vida submarina) y ODS 15 (Vida de ecosistemas terrestres). Así mismo, se requieren para el desempeño de todas las competencias clave en sostenibilidad (Murga Menoyo y Bautista Cerrro, 2019).

También el programa de la asignatura ofrece ejemplos de sistemas, desde el átomo hasta el sistema solar; aunque no son abordados como tales sino analizados únicamente en algunos de sus elementos. Esta limitación podría dar como resultado que el alumnado llegue a conocer las definiciones y quizá algunas relaciones sencillas, pero no el sistema como un conjunto dinámicoal que pertenecen todos los elementos en recíproca interacción y del que depende en su totalidad. En este caso, carecería de conceptos básicos fundamentales para comprender qué requiere el desarrollo sostenible, siendo el primero de ellos el propio concepto de sostenibilidad entendido como equilibrio dinámico (entre los contenidos del currículo, está incluido únicamentea modo de definición); el segundo concepto que se echa en falta es el de desarrollo sostenible, entendido como progreso económico y social dentro de los límites del medio natural.

Por otra parte, este déficit conceptual es muy difícil de resolver sin la comprensión previa del concepto de sistema y el desarrollo de la competencia sistémica (pensamiento sistémico) pues sólo infiriendo la dinámica conjunta que forman los recursos naturales, la economía, la sociedad $y$, en última instancia, el individuo, puede este situarse e intervenir de forma eficiente en su evolución. Un ejemplo sencillo surge al observar en el aula cómo el alumno medio domina las definiciones de atmósfera, hidrosfera, geosfera o ecosistema, pero apenas aplica estos conocímientos a interpretar los problemas de su entorno ni se ubica respecto a ellos, probablemente debido a la falta de competencia sistémica. Este déficit podría quedar enmascarado en muchas respuestas irreprochables desdeel punto de vista de las actuales rúbricas de evaluación fruto de la aplicación estricta del RD 1105/2014; sin embargo, esta falta de visión sistémica dificultaría el ejercicio del pensamiento crítico y también la implicación del alumnado en compromisos o decisiones ante las que se percibe, en el mejor de 
los casos, como un mero observador, desvinculado de emociones y responsabilidades.

En su conjunto, son contenidos propios de una alfabetización ecosocial básica y, en este sentido, componentes ineludibles de las competencias en sostenibilidad. Es en los estándares de aprendizaje, indicadores de los logros concretos que la asignatura pretende, donde con más claridad se podrá apreciar si las competencias en sostenibilidad están en el punto de mira de los procesos formativos de las asignaturas.

Respecto a los estándares de aprendizaje, en la Comunidad Autónoma de Asturias, los departa- mentos didácticos de los centros escolares elaboran los indicadores de logro a partir de los estándares de aprendizaje que se publican en la normativa vigente; quedan recogidosen forma de rúbrica, que muestra el grado de adquisición que para cada uno de ellos. Tres estándares para el bloque I, veintiuno para el bloque II, trece para el bloque III, cuatro para el bloque IV, tres para el bloque $\mathrm{V}$, cinco para el bloque $\mathrm{VI}$ y seis para el bloque VII. De ellos, como se recoge en la tabla 4 , tan solo cinco permiten evaluar explícitamente los conocimientos directamente relacionados con los Objetivos de Desarrollo Sostenible.

Ta bla 4. Biología y Geología, $1^{\circ}$ de ESO: estándares de aprendizaje afines a los ODS

\begin{tabular}{|l|}
\hline ESTÁNDARES DE APRENDIZAJE EVALUABLES \\
\hline BLOQUE II: La Tierra y el Universo \\
II.11- Reconoce la importancia del uso responsable y la gestión sostenible de los \\
recursos minerales. \\
II.15- Relaciona la contaminación ambiental con el deterioro del medio ambiente, \\
proponiendo acciones y hábitos que contribuyan a su solución. \\
II.19-Comprende el significado de gestión sostenible del agua dulce, enumerando \\
medidas concretasque colaboren en esa gestión. \\
\hline BLOQUE VI- Los ecosistemas \\
VI.3- Selecciona acciones que previenen la destrucción del medioam biente. \\
VI.5- Reconoce la fragilidad del suelo y valora la necesidad de protegerlo. \\
Fuente: Real Decreto 1105/2014, de 26 de diciembre por el que se establece el currículo básico de la Educación \\
Secundaria Obligatoria y del Bachillerato (2014).
\end{tabular}

\subsection{Capacidades que la asignatura forma relacionadas con las competencias en sostenibilidad}

Desde el punto de vista de las capacidades que el proceso formativo busca desarrollar en la asignatura de Biología y Geología de $1^{\circ}$ de la ESO, se presentan a continuación aquellas que contribuyen a robustecer las competencias en sostenibilidad por incluir elementos que conforman las competencias clásicas, como se indicaba en la tabla 3 , en este caso oficialmente prescritas para su formación en el marco de ambas disciplinas. A. Competencia ensostenibilidad: pensamien-
to crítico

La tabla 5 recoge la información referida a la competencia en sostenibilidad: pensamiento crítico. El currículum plantea exigencias para formar el pensamiento del alumnado con los criterios matemáticos de orden, cantidad, espacio, forma, perspectiva, transformación e incertidumbre y de confianza en la razón (CMCT) y con autonomía intelectual para sistematizar, comunicar y transmitir nuevas ideas (CL). Este alumno que alcanza los objetivos de la asignatura es capaz de elaborar y aplicar su propio conocímiento y persistir hasta la solución de problemas en distintos contextos (CAA), tomando la iniciativa con creatividad, decisión y coraje (SIE) y aplicando con seguridad el contenido digital a sus objetivos (CD). El currículum prescribe también un marco axiológico que orienta el pensamiento crítico hacia el compromiso ético y democrático, el respeto a la normativa y a la integración social (CSC) junto con la valoración crítica de las distintas manifestaciones culturales (CEC). 
Tabla 5. Capacidades que la asignatura fortalece en el marco de las competencias clásicas del currículo básico relacionadas con la competencia pensamiento crítico

\begin{tabular}{|c|c|}
\hline COMPETENCIAS CLÁSICAS & CAPACIDADES \\
\hline C. LINGÜÍSTICA & $\begin{array}{l}\text { - Autonomía intelectual } \\
\text { - Confianza en la razón } \\
\text { - Orden y significado } \\
\text { - Sistematiza nuevas ideas } \\
\text { - Comunicación efectiva } \\
\text { - Transmisión de ideas a nuevos contextos. }\end{array}$ \\
\hline $\begin{array}{l}\text { C.MATEMATICA, CIENCIAY } \\
\text { TECNOLÓGICA }\end{array}$ & $\begin{array}{l}\text { - Orden y significado } \\
\text { - Cantidad, espacio, forma, perspectiva, transformación } \\
\text { - Interpretación y valoración de conclusionesen incertidumbre }\end{array}$ \\
\hline C.DIGITAL & $\begin{array}{l}\text { - Valoración critica de las fuentes de información } \\
\text { - Transformación de contenido digital en conocimiento útil } \\
\text { - Control de seguridad } \\
\text { - Comunicación efectiva } \\
\text { - Compromiso intelectual yético }\end{array}$ \\
\hline C. APRENDER A APRENDER & $\begin{array}{l}\text { - Motivación y confianza } \\
\text { - Planificación, persistencia y evaluación } \\
\text { - Solución de problemas a partir de las propias herramientas } \\
\text { - Transferencia a otras situaciones }\end{array}$ \\
\hline C.SOCIALES Y CÍVICAS & $\begin{array}{l}\text { - Comprom iso intelectual, integridad, humildad, justicia } \\
\text { - Compromiso ético, em patía, equidad, objetividad } \\
\text { - Códigos de conducta social y normativa. } \\
\text { - Toma de decisiones democráticas. }\end{array}$ \\
\hline $\begin{array}{l}\text { C.INICIATIVAY ESPIRITU } \\
\text { EMPRENDEDOR }\end{array}$ & $\begin{array}{l}\text { - Autonomía intelectual } \\
\text { - Confianza en la razón } \\
\text { - Compromiso ético e intelectual } \\
\text { - Coraje y decisión } \\
\text { - Creatividad } \\
\end{array}$ \\
\hline $\begin{array}{l}\text { C.CONCIENCIAY } \\
\text { EXPRESIONES CULTURALES }\end{array}$ & $\begin{array}{l}\text { - Valoración crítica de las distintas manifestaciones culturales } \\
\text { incluidas las cotidianas de su propia vida. }\end{array}$ \\
\hline
\end{tabular}

Fuente: Elaboración propia

B. Competencia en sostenibilidad: pensamiento sistémico

Esta asignatura ofrece muchos ejemplos de sistemas naturales que no llegan a ser interpretados como tales al no haber introducido previamente el concepto de sistema. La tabla 6 muestra que las competencias clásicas sólo ofrecen una aproximación atomizada de los elementos del sistema y de algunas relaciones. En reciprocidad, la falta de pensamiento sistémico afecta negativamente al desarrollo de otras competencias. El currículum puede capacitar al alumnado para conceptualizar y expresar vínculos con el entorno $(\mathrm{CL})$, pero no cubre la totalidad de los aspectos a tener en cuenta. Asimismo, el alumnado interpreta perspectivas, relaciones, transformaciones, resultados e incertidumbres
(CMCT) pero, al no estudiar el concepto de sistema las interpretaciones son parciales. El entorno digital es reticular, atemporal y multidimensional (CD), características del pensamiento sistémico, pero se contemplan desde un punto de vista meramente instrumental. Igualmente, la auto capacidad resolutiva (CAA) y la interpretación de problemas en su contexto (SIE) quedan mermadas por la falta de competencia sistémica. Por último, los elementos de ciudadanía, integración social y empatía con otras situaciones personales (CSC) así como el respeto a las manifestaciones culturales y artísticas (CEC), nutren con ejemplos al pensamiento sistémico, pero es la carencia de este la que lastra el posicionamiento y la acción respecto a estos problemas sociales. 
Tabla 6. Capacidades que la asignatura fortalece en el marco de las competencias clásicas del currículo básico relacionadas con la competencia pensamiento sistémico

\begin{tabular}{|c|c|}
\hline COMPETENCIAS CLÁSICAS & CAPACIDADES \\
\hline C. LINGÜÍSTICA & $\begin{array}{l}\text { - Conceptualización y expresión de los vínculos establecidos con } \\
\text { los demás y en el contexto } \\
\text { - Afecta a competencia lingüística } \\
\text { - Solo expresa lo que comprende previamente. }\end{array}$ \\
\hline $\begin{array}{l}\text { C.MATEMATICA, CIENCIAY } \\
\text { TECNOLÓGICA }\end{array}$ & $\begin{array}{l}\text { - Ubica e interpreta perspectivas, relaciones, transformaciones y } \\
\text { resultados y asume la incertidumbre. } \\
\text { - No trabaja con el concepto de sistema } \\
\text { - No se integra a sí mismo en el sistema porque no lo interpreta } \\
\text { como tal. }\end{array}$ \\
\hline C.DIGITAL & $\begin{array}{l}\text { - Espacios multidimensionales } \\
\text { - Concepto reticular } \\
\text { - Atemporalidad } \\
\text { - Evolución continúa de variables } \\
\text { - Insuficiente por falta de competencia sistémica } \\
\end{array}$ \\
\hline C. APRENDER A APRENDER & $\begin{array}{l}\text { - Es la competencia sistémica la que aporta situación, variables y } \\
\text { entorno a la necesidad de aprender. }\end{array}$ \\
\hline C.SOCIALES Y CIVICAS & $\begin{array}{l}\text { - Ciudadanía e integración social. } \\
\text { - Empatía con otras situaciones personales y colectivas (visión } \\
\text { global de situación). } \\
\text { - Carencia de visiónglobal, déficit de empatía al no saber ubicarse } \\
\text { dentro de la situación. }\end{array}$ \\
\hline $\begin{array}{l}\text { C.INICIATIVAY ESPÍRITU } \\
\text { EMPRENDEDOR }\end{array}$ & $\begin{array}{l}\text { - Interpretación de problemas en su contexto } \\
\text { - Deficiente por falta de competencia sistémica }\end{array}$ \\
\hline $\begin{array}{l}\text { C.CONCIENCIAY } \\
\text { EXPRESIONES CULTURALES }\end{array}$ & $\begin{array}{l}\text { - Deficiente comprensión de las expresiones culturales y } \\
\text { artísticas como adaptación al contexto por falta de la } \\
\text { competencia sistémica }\end{array}$ \\
\hline
\end{tabular}

Fuente: Elaboración propia

C. Competencia en sostenibilidad: "toma de decisiones colaborativa"

La tabla 7 refleja los elementos básicos que las competencias clásicas aportan para la toma de decisiones colaborativa. Con el estudio de la asignatura el alumnado puede adquirir la capacidad de relacionar conceptos y procedimientos, modelos, previsiones y riesgos e interpretar datos en situaciones concretas (CMCT), a través de entornos digitales (CD). También podrá organizar y gestionar el propio aprendizaje para contribuir a la tarea grupal desde la responsa- bilidad individual (CAA); y, además, comunicar y negociar (CL) y asumir la tarea de forma autónoma e innovadora (SIE). Asimismo, dicha participación grupal se realiza desde el reconocimiento de la identidad individual y social y la ciudadanía democrática (CSC) y el respeto a las distintas manifestaciones culturales y artísticas (CEC). Sin embargo, no constan los elementos de liderazgo, empatía y asertividad indispensables para el trabajo en grupo; ni se muestran evidencias de la integración en un grupo de trabajo real, el tipo de participación o los resultados más allá del entorno del aula.

Tabla 7. Capacidades que la asignatura fortalece en el marco de las competencias clásicas relacionadas con la competencia toma de decisiones colaborativa

\begin{tabular}{|l|l|}
\hline \multicolumn{1}{|c|}{ COMPETENCIAS CLÁSICAS } & \multicolumn{1}{c|}{ CAPACIDADES } \\
\hline C. LINGÜÍSTICA & $\begin{array}{l}\text { - Comprender, representar y expresar un problema en distintas } \\
\text { situaciones } \\
\text { - Expresiones de liderazgo, de negociación }\end{array}$ \\
\hline $\begin{array}{l}\text { C.MATEMATICA, CIENCIA Y Y } \\
\text { TECNOLÓGICA }\end{array}$ & $\begin{array}{l}\text { - Relaciona conceptos y procedimientos en la resolución de } \\
\text { tareas. } \\
\text { - Aporta interpretación de datos en situacionesconcretas }\end{array}$ \\
\hline
\end{tabular}




\begin{tabular}{|c|c|}
\hline & $\begin{array}{l}\text { - Aporta modelos, previsiones y riesgos. } \\
\text { - En la práctica la gestión de la incertidumbre y la asunción de } \\
\text { riesgos es deficiente. }\end{array}$ \\
\hline C.DIGITAL & $\begin{array}{l}\text { - Comunicación instantánea y continúales en todos los contextos } \\
\text { y situaciones. } \\
\text { - Seguimiento de resultados } \\
\text { - Influencia y negociación }\end{array}$ \\
\hline C. APRENDER A APRENDER & $\begin{array}{l}\text { - Organizar y gestionar el propio aprendizaje para contribuir a la } \\
\text { tarea grupal } \\
\text { - Planificación, motivación, persistencia y comunicación }\end{array}$ \\
\hline C. SOCIALES Y CIVICAS & $\begin{array}{l}\text { - Identificación individual y social. } \\
\text { - Participación ciudadana democrática. } \\
\text { - Empatía con otros valores, creencias y situaciones individuales } \\
\text { y colectivas. } \\
\text { - Asertividad } \\
\text { - Comprensión y respeto a la normativa. }\end{array}$ \\
\hline $\begin{array}{l}\text { C.INICIATIVA Y Y ESPÍRITU } \\
\text { EMPRENDEDOR }\end{array}$ & $\begin{array}{l}\text { - Autonomía, innovacióno aportes al grupo. } \\
\text { - Negociación. } \\
\text { - Asunción de riesgos e incertidumbre. }\end{array}$ \\
\hline $\begin{array}{l}\text { C.CONCIENCIAY EXPRESIONES } \\
\text { CULTURALES }\end{array}$ & $\begin{array}{l}\text { - Respeto a distintas manifestaciones culturales y artísticas } \\
\text { - Comprensión de su significado. }\end{array}$ \\
\hline
\end{tabular}

Fuente: Elaboración propia

D. Competencia en sostenibilidad: "responsabilidad con las generaciones presentesyfuturas"

La tabla 8 recoge las capacidades básicas necesarias para la competencia de responsabilidad con las generaciones presentes y futuras. Facilitan que el alumnado se comunique correctamente con personas de distintas generaciones y contextos (CL), sea capaz de razonamiento automático que interpreta datos y aporta solu- ciones (CMCT) en espacios reales o virtuales (CD), desde la iniciativa y el emprendimiento (SIE), la plena integración social (CSC) y el respeto a las diferencias (CEC). Pero una vez más, entre los elementos del currículum no consta la exigencia de evidencias de compasión, ni de comprender los efectos que nuestro estilo de vida causa en las vidas de otras personas, ni la demostración de un compromiso personal y mantenido con el desarrollo sostenible desde la adopción de nuevos estilos de Vida compatibles con la sostenibilidad.

Tabla 8. Capacidades que la asignatura fortalece en las competencias clásicas relacionadas con la responsabilidad con las generaciones presentes y futuras

\begin{tabular}{|l|l|}
\hline \multicolumn{1}{|c|}{$\begin{array}{c}\text { COMPETENCIAS } \\
\text { CLÁSICAS }\end{array}$} & \multicolumn{1}{c|}{ CAPACIDADES } \\
\hline C. LINGÜÍSTICA & $\begin{array}{l}\text { - Comunicación entre la propia generación y otras generaciones vivas en } \\
\text { distintas situaciones y contextos. }\end{array}$ \\
\hline $\begin{array}{l}\text { C.MATEMATICA, } \\
\text { CIENCIAY } \\
\text { TECNOLÓGICA }\end{array}$ & $\begin{array}{l}\text { - Concreción y ubicación de datos, de problemas y sus soluciones. } \\
\text { - Previsión de futuros escenarios en su entorno y en otros entornos. }\end{array}$ \\
\hline C.DIGITAL & $\begin{array}{l}\text { - Comunicación sistemática e inmediata con distintas generaciones vivas } \\
\text { en distintos lugares. } \\
\text { - Atemporalidad } \\
\text { - Aproximación a emociones. }\end{array}$ \\
\hline C.APRENDERA & $\begin{array}{l}\text { - Motivación para analizar circunstancias e intereses de personas de } \\
\text { distintas generaciones de diferentes entornos culturales. }\end{array}$ \\
\hline CRRENDER & $\begin{array}{l}\text { - Compromiso social con personas de otras generaciones yotras culturas } \\
\text { mediante la formación en valores. }\end{array}$ \\
& $\begin{array}{l}\text {-Valoración crítica y respeto de la normativa vigentey de distintos códigos } \\
\text { de conducta. }\end{array}$ \\
\hline
\end{tabular}




\begin{tabular}{|l|l|}
\hline C.INICIATIVAY & $\begin{array}{l}\text { - Iniciativa, innovación, procedimiento y decisión para abordar problemas } \\
\text { de distintas generaciones. } \\
\text { - Autonomía intelectual y ética para asociarse, emprendery trabajar con } \\
\text { EMPRENDEDOR }\end{array}$ \\
\hline $\begin{array}{l}\text { C.CONC tipo de personas. } \\
\text { EXPRESIONCIAY } \\
\text { CULTURALES }\end{array}$ & $\begin{array}{l}\text { - Respeto a distintas manifestaciones culturales y artísticas } \\
\text { - Comprensión de su significado. }\end{array}$ \\
\hline
\end{tabular}

Fuente: elaboración propia

\subsubsection{Contribución de la asignatura Biología y Geología de $1^{\circ}$ de ESO a la formación de competencias para los ODS}

Iniciábamos el estudio preguntándonos si el actual currículo oficial de la educación secundaria obligatoria facilita la formación de los conocimientos, valores, actitudes u otros elementos que conforman las competencias en sostenibilidad demandadas por Unesco. Y focalizábamos la atención en cuatro competencias clave en sostenibilidad, con la intención de identificar en los estándares de aprendizaje establecidos por la normativa vigente evidencias para una respuesta positiva. En la tabla 9 se presentan de forma sintética los resultados del análisis.

Las cuatro competencias analizadas abarcan los distintos dominios de la formación integral del alumnado. En primer lugar, el pensamiento crítico se desarrolla a partir de la autonomía intelectual y el compromiso ético; permite interpretar de forma veraz pensamientos y hechos, someterlos a valoración ética y saber actuar con decisión y coraje para cambiar la tendencia de los factores que contribuyen a situaciones desfavorables en distintos contextos.

A la vez, esa misma confianza en la razón implica la humildad para reconocer las propias limitaciones al interpretar hechos y contextos, y evitar la tendencia a infravalorar otras opiniones y culturas en favor de las propias. La asignatura contribuye a ello a través de la comprensión del método científicoy de su aplicación en los distintos bloques de conocimiento. Las características de la ciencia revelan las dificultades que ha de vencer el avance del conocimiento, sujeto siempre a nuevas evidencias, al igual que señalan fronteras para la capacidad humana.

En segundo lugar, la competencia sistémica requiere el desarrollo de los componentes de organización y conceptualización reticular, así como sus relaciones, dinámica interna, interacciones y resultados (Morin, 2004)y trascender el pensamiento lineal para comprender la complejidad de los sistemas naturales y sociales que facilitan la respuesta conjunta y cambiante de adaptación a sus entornos. La asignatura ofrece muchos ejemplos a lo largo de sus unidades, en particular la diversificación de las especies a partir de un antepasado común; sin embargo, no facilita al alumnado suficientes herramientas de interpretación que le permita comprenderlas como tales sistemas.

En tercer lugar, la competencia de toma de decisiones colaborativa requiere desarrollar los elementos de responsabilidad personal, empatía, asertividad e integración necesarios para el trabajo en grupo orientado a alcanzar objetivos comunes en distintos contextos (UNESCO, 2018). A este respecto, alumnos y alumnas deben reconocerse a sí mismos como parte interesada en la resolución final, e identificarse como miembros de un equipo que se verá afectado por su compromiso y sus aportaciones. El currículum de esta asignatura integra un bloque destinado a la realización de un Proyecto de investigación en el que se trabajan todos los componentes que forman dicha competencia, si bien el documento oficial no exige la transferencia de resultados del aula a la vida real.

Finalmente, la competencia de responsabilidad con las generaciones presentes y futuras exige compromiso intelectual, ético y social para establecer relacionesjustas entre todas las generaciones presentes y preservar los bienes materiales e inmateriales para las generaciones futuras (UNESCO, 2014). El alumnadotiene quecom-prender su propia influencia en este propósito, asumiendo que ninguna de sus decisiones carece de efecto sobre el entorno. La asignatura introduce en las consecuencias de algunoscom portamientos humanos sobre el medio ambiente; en concreto la contaminación atmosférica, el efecto invernadero, la destrucción de la capa de ozono, la contaminación hídrica en aguas de uso industrial, agroganadero o doméstico, de la actividad minera y la contaminación de suelos sobre el medio ambiente, pero no exige que el alumnado pase del aprendizaje teórico a un planteamiento de solución basado en la vida real. 
Tabla 9. Elementos: Componentes y capacidades* de las competencias para el desarrollo sostenible, contextualizadas en la asignatura de Biología y Geología de $1^{\circ}$ ESO**

\begin{tabular}{|c|c|c|}
\hline COMPETENCIA & COMPONENTES & $\begin{array}{c}\text { CAPACIDADES } \\
\text { (ESTÁNDARES DE APRENDIZAJE) }\end{array}$ \\
\hline $\begin{array}{l}\text { PENSAMIENTO CRITICO } \\
\text { (1) }\end{array}$ & $\begin{array}{l}\text { - Compromiso } \\
\text { intelectual, integridad, } \\
\text { humildad yjusticia } \\
\text { - Compromiso ético, } \\
\text { empatía, equidad, } \\
\text { objetividad } \\
\text { - Autonomía intelectual } \\
\text { - Confianza en la razón } \\
\text { - Coraje y decisión } \\
\text { - Creatividad }\end{array}$ & $\begin{array}{l}\text { - Sistematizar las nuevas ideas } \\
\text { - Comprender pensamiento incompleto, } \\
\text { subjetivo, sesgado o hipotético } \\
\text { - Controlar su aprendizaje, preguntas } \\
\text { esenciales, recopilación y evaluación de } \\
\text { información relevante } \\
\text { - Superar egocentrismo y sociocentrismo y } \\
\text { limitaciones propias } \\
\text { - Comunicación efectiva } \\
\text { - Transmisión de ideas a nuevos contextos } \\
\text { - Tomar decisiones, propuestas de mejora }\end{array}$ \\
\hline $\begin{array}{l}\text { PENSAMIENTO } \\
\text { SISTÉMICO (2) (3) }\end{array}$ & $\begin{array}{l}\text { - Organización } \\
\text { - Dirección y control } \\
\text { - Integración } \\
\text { - Teleología } \\
\text { - Conceptualización } \\
\text { reticular } \\
\text { - Conceptualización } \\
\text { hologramática }\end{array}$ & $\begin{array}{l}\text { - Comprender la complejidad biológica de los } \\
\text { sistemas naturales. } \\
\text { - Comprender la complejidad cultural y social } \\
\text { - Diferenciar e integrar los diferentes campos } \\
\text { de la cultura y la naturaleza } \\
\text { - Establecer analogías entre distintos sistemas } \\
\text { y distintos contextos. } \\
\text { - Trascender la linealidad causa-efecto } \\
\text { - Percibir la composición, vinculación, } \\
\text { estructura relacionaly procesos de asuntos de } \\
\text { interés. }\end{array}$ \\
\hline $\begin{array}{l}\text { TOMA DE DECISIONES } \\
\text { COLABORATIVA } \\
\text { (4) (5) }\end{array}$ & $\begin{array}{l}\text { - Responsabilidad } \\
\text { individual y grupal } \\
\text { - Planificación, } \\
\text { coordinación y } \\
\text { cooperación } \\
\text { - Liderazgo y autoridad } \\
\text { compartida: empatía y } \\
\text { asertividad. } \\
\text { - Seguimiento y } \\
\text { retroalimentación } \\
\text { - Riesgo e incertidumbre }\end{array}$ & $\begin{array}{l}\text { - Integrarse con éxito en un grupo de trabajo } \\
\text { complejo a través de objetivos comunes } \\
\text { - Comprender y representar el contenido del } \\
\text { problema, aplicando estrategias para } \\
\text { resolverlo y mecanismos de regulación en } \\
\text { compromiso con otros miembros del grupo } \\
\text { - Responsabilizarse individualmente de las } \\
\text { decisiones tomadas de forma conjunta. } \\
\text { - Asumiry gestionar la peor situación posible }\end{array}$ \\
\hline $\begin{array}{l}\text { RESPONSABILIDAD } \\
\text { CON LAS } \\
\text { GENERACIONES } \\
\text { PRESENTESY FUTURAS } \\
\text { (6) }\end{array}$ & $\begin{array}{l}\text { - Compasión } \\
\text { - Compromiso ético } \\
\text { - Compromiso social } \\
\text { - Pensamiento } \\
\text { anticipatorio } \\
\text { - Pensamiento } \\
\text { sincrónico y diacrónico } \\
\text { - Responsabilidad } \\
\text { universal }\end{array}$ & $\begin{array}{l}\text { - Comprender los efectos que, a medio ylargo } \\
\text { plazo, tienen los comportamientos } \\
\text { individuales sobre los usos y costumbres } \\
\text { sociales de la propia comunidad y de otras. } \\
\text {-Comprender las consecuencias de los } \\
\text { comportamientos individuales y colectivos } \\
\text { sobre las condiciones biológicas necesarias } \\
\text { para la vida presente y futura. } \\
\text {-Establecer relaciones justas con personas de } \\
\text { su generación y de otras } \\
\text {-Contribuir al cambio por la sostenibilidad, } \\
\text { adoptando estilos de vida sostenibles. }\end{array}$ \\
\hline
\end{tabular}

Fuente: Elaboración propia a partir de autores varios: (1) Wiek, Withycombe \& Redman, 2011; (2) Morin, 2010; (3) Herrera, 2008; (4) OCDE, 2015; (5) UNESCO, 2018; (6) UNESCO,2014

* Las capacidades (evidencias de adquisición de las competencias) son denominadas como "estándares de aprendizaje" según la terminología oficial (RD 1105/2014, de 26 de diciembre).

** La categorización de los elementos de las competencias en "componentes" y "capacidades" obedece al criterio de considerar a los primeros como los atributos que conforman la competencia y que deben ser específicamente formados en el aula, mientras que bajo las capacidades se recogen las evidencias de adquisición de estas por parte del alumnado. 


\section{CONCLUSIONES}

Desde el punto de vista de la educación para la sostenibilidad, enfoque que promueve la UNESCO, explícitamente, en la meta 4.7 de la Agenda 2030 (ONU, 2015, p.20), la asignatura de Biología y Geología de $1^{\circ} \mathrm{ESO}$, tal como está planteada en el currículo básico oficial, facilita la adquisición de conocimientos y capacidadesque conforman cuatro competencias clave para la sostenibilidad: pensamiento crítico, pensamiento sistémico, toma de decisiones colaborativa y responsabilidad con las generaciones presentes y futuras. Lo hace, sin embargo, de forma insuficiente, dejando lagunas por atender tanto en el plano conceptual como en los estándares de aprendizaje, que no recogen específicamente evidencias prácticas de compromiso con el desarrollo sostenible.

Cabe considerar puntos fuertes del marco curricular de la asignatura:

- Un conjunto de contenidos que proporcionan conocimientos sobre el medio natural, necesarios para comprender el enfoque del desarrollo sostenible.

- El desarrollo del pensamiento crítico.

- Las capacidades básicas necesarias, aunque no suficientes, para la toma de decisiones colaborativas y la responsabilidad con las generaciones presentes y futuras.

- Un tímido acercamiento al desarrollo sostenible desde el plano teórico

Las principales debilidades detectadas:

- Lagunas conceptuales que dificultan la comprensión de los ODS, tales como la ausencia de los conceptos de sistema, pobreza, cambio climático o desarrollo sostenible.

- Escasa atención al desarrollo del pensamiento sistémico

- Descuido a la dimensión emocional de la educación, desaprovechando la potencialidad del entorno para generar vínculos alumnado-naturaleza que motiven el compromiso activo por la sostenibilidad.
- Ausencia de estándares de aprendizaje para evaluar el compromiso activo del alumnado ante las problemáticas ecológicas, económicas y sociales del entorno.

- Falta de exigencia de compromiso con el cambio hacia un estilo de vidasostenible.

Las principales limitaciones que ha encontrado el trabajo están en que prácticamente todos los estándares de aprendizaje cum plen su misión de calibrar la adquisición de competencias en el plano teórico, con lo cual sirven para evaluar conocimientos sobre el medio natural pero no exigen que este conocimiento traspase los límites del aula mediante su puesta en práctica e implicación del alumnado en asuntos reales del entorno. Tampoco se exigen evidencias a partir de ejemplos del propio estilo de vida que demuestren compromiso con los Objetivos de Desarrollo Sostenible.

El estudio viene a confirmar el planteamiento retórico que del enfoque por competencias se realiza en la normativa vigente. Si bien se reconoce en ella la necesidad de formar competencias -en línea con las más actuales tendencias internacionales- el énfasis del proceso formativo se sitúa en la adquisición de contenidos disciplinares, como resulta notorio al consultar los estándares que se establecen para la evaluación.

Sin embargo, la formación de competencias, entre ellas las competencias en sostenibilidad, requiere un paso más. Es preciso relacionar los contenidos disciplinares de la asignatura, Ios temas del programa, con las problemáticas que abordan los ODS y, a través de esos contenidosy de las metodologías de aula facilitar al alu mnado la adquisición del conjunto de los numerosos elementos (valores, habilidades, hábitos, capacidades, actitudes, etc.) que, en recíproca interacción, tienen como efecto un comportamiento que evidencie sus competencias en sostenibilidad. Esta es una gran tarea pendiente para el profesorado de la educación secundaria, cuya contribución como agente de cambio a los objetivos de la Agenda 2030 es una necesidad social ineludible. 


\section{REFERENCIAS BIBLIOGRÁFICAS}

- Comisión Europea (2015). Science Education for Responsible Citizenship.

http://ec.europa.eu/research/swafs/pdf/pub_scie nce_education/KI-NA-26-893-EN-N.pdf.

- Consejo de la Unión Europea (2018). Recomendación del Consejo relativa a las competencias clave para el aprendizaje permanente (Texto pertinente a efectos del EEE). 2018/C 189/01. Diario Oficial de la Unión Europea. https://eur-lex.europa.eu/legalcontent/ES/TXT/PDF/?uri=CEL EX:3 2018H 0604(0 1) \& from $=S V$.

- Cortes Generales (2013, 9 de diciembre). Ley Orgánica 8/2013, de 9 de diciembre, para la Mejora de la Calidad Educativa. Boletín Oficial del Estado de 10 de diciembre de 2013.

https://www.boe.es/buscar/pdf/2013/BOE-A2013-12886-consolidado.pdf.

- Herrera R. (2008). Sistema y lo sistémico en el pensamiento contemporáneo. Ingeniería: Revista de la Universidad de Costa Rica, 1(2), 37-52. https://revistas.ucr.ac.cr/index.php/ingeni eria/art icle/view/7745/7409

- Kozlowski, S.W.J. \& Ilgen, D.R. (2006). Enhancing the efectiveness of work groups and teams. Psychological Science in the Public Interest, 7(3), 77-124. DOI: https://doi.org/10.1111/j.15291006.2006.00030.x.

- Ministerio de Educación, Cultura y Deporte (2014, 26 de diciembre). Real Decreto 1105/2014, de 26 de diciembre por el que se establece el currículo básico de la Educación Secundaria Obligatoria y del Bachillerato. Boletín Oficial del Estado, nํ3, de 3 de enero de 2015.

https://www.boe.es/boe/dias/2015/01/03/pdfs/ BOE-A-2015-37.pdf.

- Ministerio de Educación, Cultura y Deporte. (2015, 21 de enero). Orden ECD/65/2015, de 21 de enero por la que se describen las relaciones entre las competencias, los contenidos y los criterios de evaluación de la educación primaria, la educación secundaria obligatoria y el bachillerato. Boletín Oficial del Estado, nº. 25, de 29 de enero de 2015. https://www.boe.es/eli/es/o/2015/01/21/ec d65 /dof/spa/pdf.

- Morín, E. (2004). Introducción al pensamiento complejo. Editorial Gedisa.

- Morin E. (2010). Anotaciones para un nuevo Emilio: transmisión sistémica del conocimiento. Signo y Pensamiento, XXIX(56), 42-49. https://www.redalyc.org/articulo.oa?id $=8601934$ 8002.

- Murga Menoyo, M.A. (2015): Competencias para el desarrollo sostenible: las capacidades, actitudes y valores meta de la educación en el marco de la Agenda global post-2015. Foro de Educación, 13(19), 55-83. DOI: http://dx.doi.org/10.14516/fde.2015.013.019.004.

- Murga Menoyo, M. A. y Bautista Cerro, M.J. (Eds.) (2019). Guía PRADO. Sostenibilizar el currículo de las Educación Secundaria. UNED. http://espacio.uned.es/fez/view/bibliun ed:c atedraUnesco EADS-Libros-Amurga0001.

- Novo, M., Mandón, M.J. y Marpegán, C.M. (2002). EI enfoque sistémico: su dimensión educativa. UNED.

- OCDE (2015). PISA 2015. Assessment and Analytical Framework. Science, Reading, Mathematic, Financial Literacy and Collaborative Problem Solving. https://www.oecdilibrary.org/docserver/9789264281820en.pdf? expires $=1602704494 \& i d=i d \& a c c n a m e=g u$ est\&checksum=6D5AB921F75C4F5257AE1B DE0 2 $685 \mathrm{E} 46$

- OCDE (2005). La definición y selección de competencias clave. Resumen ejecutivo. https://www.deseco.ch/bfs/deseco/en/in dex/03/ 02.parsys.78532.downloadList.94248.Downlo adFil e.tmp/2005.dscexecutivesummary.sp.pdf.

- ONU (2015, 25 de septiembre). Resolución 2015.A/RES/70/1. Transformar nuestro mundo: la Agenda 2030 para el Desarrollo Sostenible. https://unctad.org/system/files/officialdocument/ares70d1_es.pdf.

- Parlamento europeo y Consejo de la Unión Europea (2006). Recomendación del Parlamento Europeo y del Consejo sobre las competencias clave para el aprendizaje permanente 2006/962/CE. Diario Oficial de la Unión Europea. https://eurlex.europa.eu/legalcontent/ES/TXT/HTML/?uri=CELEX:32 006 H0962 $\&$ from $=\mathrm{LV}$.

- PNUD (2018). Índices e indicadores de Desarrollo Humano. Actualización estadística de 2018. Programa de las Naciones Unidas para el Desarrollo. http://hdr.undp.org/sites/default/files /2018_hum an_development_statistical_update_es.pdf.

- Richard P. y Elder L. (2005). Fundación para el pensamiento crítico. www.criticalthinking.org.

- Rychen D.S. y Salganik L.H. (Eds.). (2004). Definir y seleccionar las competencias fundamentales para la vida. Fondo de Cultura Económica.

- Rychen D.S., Salganik L.H., Moser, U. y Konstant, J.W. (2000). Proyectos sobre Competencias en el Contexto de la OCDE Análisis de base teórica y conceptual. https://www.deseco.ch/bfs/deseco/en/in dex/03/ 
02.parsys.59225.downloadList.58329.DownloadFil e.tmp/1999.proyectoscompetencias.pdf.

- UNESCO (2014). Hoja de ruta para la ejecución del programa de acción mundial de educación para el desarrollo sostenible.

https://unesdoc.unesco.org/ark:/48223/pf000 023 0514_spa.

- UNESCO (2017). Educación para los Objetivos de Desarrollo Sostenible: objetivos de aprendizaje. https://unesdoc.unesco.org/ark:/48223/pf000 025 2423.

- UNESCO (2018). Issues and trends in education for sustainable development.

https://unesdoc.unesco.org/ark:/48223/p f000 026 1445

- Wiek, A., Withycombe, L. \& Redman, C. L. (2011). Key competencies in sustainability: a reference framework for academic program development. Sustainability Science, 6(2), 203-218. DOI: https://doi.org/10.1007/s11625-011-0132-6

- WWF (2020). Living Planet Report 2020 Bending the curve of biodiversity loss. WWF.

https://www.footprintnetwork.org/content/uploa ds/2020/09/LPR2020-Full-report-lo-res.pdf. 